\title{
High Quality GSM Voice based Analysis of the Prominent Routing Protocols of MANET
}

\author{
Meenakshi Sharma \\ M.Tech Scholar \\ SBSSTC, \\ Ferozepur
}

\author{
Navdeep Kaur \\ A.P-ECE \\ SBSSTC, \\ Ferozepur
}

\author{
Gurjeevan Singh \\ DIC-ECE \\ SBSSTC(P.W.), \\ Ferozepur
}

\begin{abstract}
MANETs represent complex distributed systems which are comprised of free moving mobile nodes which use dynamic topology and are infrastructure less networks. The prime aim of this paper is to present the complete study on the performance and behavior of reactive, proactive and hybrid approach in MANETs. To evaluate the performance of these protocols different scenarios with varying nodes have been simulated using the NS-2 simulation tool. There are some performance metrics which are used to describe the performance of these given routing protocols e.g. number of packets sent, number of packets received, number of packets dropped, Normalized routing Load, Network Overload etc.
\end{abstract}

\section{Keywords}

\section{AODV,DSDV,MANET,NRL,ZRP}

\section{INTRODUCTION TO MANETS}

A mobile adhoc network is a collection of independent mobile nodes like mobile phones, laptops, PCs etc. that can communicate with each other via radio waves .The mobile nodes that are in coverage range of each other can directly communicate with each other, whereas the other need the aid of intermediate nodes to route their packets[1]. Adhoc wireless network must be capable to self-organize and selfconfigure due to the fact that the mobile structure is changing all the time. MANETs are used in many fields like emergency relief scenarios, public meetings, virtual classrooms etc[4]. They also play an important role in civilian forums such as conferences, conventional centers and electronic classrooms. The features of MANETs are highly dynamic topology, Bandwidth constrained links, limited physical security and energy constrained nodes[2].

\section{TYPES OF ROUTING PROTOCOLS IN MANETS}

Routing Protocols can be classified in three categories: Reactive(or On-Demand), Proactive(or Table-Driven), Hybrid(or Zone-based).

\subsection{Proactive routing protocols}

These type of protocols are table based because they maintain table of connected nodes to transmit data from one node to another and each node share its table with another nodes. They are called proactive because the routing information is maintained by them even before it is required. Due to the presence of entries for each and every node in table, it causes more overhead in routing table which leads to more bandwidth consumption .So, these protocols are not suitable for larger networks. e.g. Destination Sequenced Distance Vector(DSDV),Cluster-Head Gateway Switch Routing(CGSR), Wireless Routing protocol(WRP) etc[2,9].

\subsection{Reactive Routing Protocols}

It is called an on-demand routing protocol because routes are only established whenever required rather than maintaining a complete list of routing information all the time. Whenever a node requires a route to destination a route discovery is initiated within the network. This process is completed once a route is found. Once a route is established, it is maintained by a route maintenance procedure till the destination becomes unreachable. e.g. Dynamic Source Routing(DSR), Adhoc OnDemand Distance Vector(AODV), Temporally ordered routing algorithm(TORA) etc[2,9].

\subsection{Hybrid Routing Protocols}

To increase the scalability and efficiency of routing protocols, hybrid routing protocol combines the characteristics of both reactive and proactive routing protocols. These protocols are based on concept of zones i.e. whole network is divided into number of zones e.g. Zone Routing Protocol(ZRP)[2,9].

\section{SIMULATION SETUP}

Simulation work has been performed on different type of protocols having 75,100,125 and 150 nodes. The simulation area taken is $5000 \mathrm{mx} 5000 \mathrm{~m}$. For simulation of AODV,DSDV and ZRP, NS-2 simulator has been used.

Table 1.Simulation setup

\begin{tabular}{|l|l|}
\hline Protocols & $\begin{array}{l}\text { AODV(Reactive), DSDV(Proactive) } \\
\text { and ZRP(Hybrid) }\end{array}$ \\
\hline Simulator & NS-2 \\
\hline Nodes & $75,100,125$ and 150 \\
\hline Simulation Area & $5000 \mathrm{~m} * 5000 \mathrm{~m}$ \\
\hline Packet Size & $1 \mathrm{kbps}$ \\
\hline Traffic Type & High Quality GSM voice \\
\hline Simulation Time & $1000 \mathrm{sec}$ \\
\hline $\begin{array}{l}\text { Physical } \\
\text { Standard }\end{array}$ & $802.11 \mathrm{~b}$ \\
\hline
\end{tabular}

\section{PERFORMANCE METRICS}

The performance metrics like number of packets sent, number of packets received, number of packets dropped, normalized routing load and network overload are chosen in order to evaluate the performance of proactive, reactive and hybrid routing protocols: 


\subsection{Number of packets sent}

During transmission of data in virtual adhoc network routing traffic is sent by all wireless nodes. In other words it shows that how much traffic is sent from source node to destination with the help of intermediate nodes in a particular simulation area using MANET routing protocols.

\subsection{Number of packets received}

It is basically number of packets received by the destination node from the source node via intermediate nodes for specified simulation area. The number of packets received can be calculated by subtracting number of packets lost and number of packets dropped from the number of packets sent. Mathematically, it can be represented as,

$\mathrm{P}_{\mathrm{r}}=\mathrm{P}_{\mathrm{s}}-\left(\mathrm{P}_{\mathrm{l}}+\mathrm{P}_{\mathrm{d}}\right)$

where $P_{r}$ is number of received packets,

$\mathrm{P}_{\mathrm{S}}$ is number of sent packets,

$P_{1}$ is number of packets lost and

$P_{d}$ is number of packets dropped.

\subsection{Number of packets dropped}

When one or more packets sent from source to the destination fail to reach their destination and are dropped by the routers in between the transmission due to any error condition in the network, they are considered as dropped packets.

\subsection{Normalized routing Load}

It can be defined as the total number of control packets that has been transported per data packet and is calculated by dividing the total number of control packets sent by the total number of data packets received by destination[9].

\subsection{Network Overload}

In wireless mobile adhoc networks, when there is congestion in the network due outsized number of nodes which are sending and receiving data beyond the limit of its communication area, this is known as network overload.

\section{RESULTS AND OBSERVATIONS}

This section shows the comparison between various capabilities of the proactive, reactive and hybrid routing protocols by having a brief overview of their performance parameters. The simulation results can e shown in the form of graphs shown below:

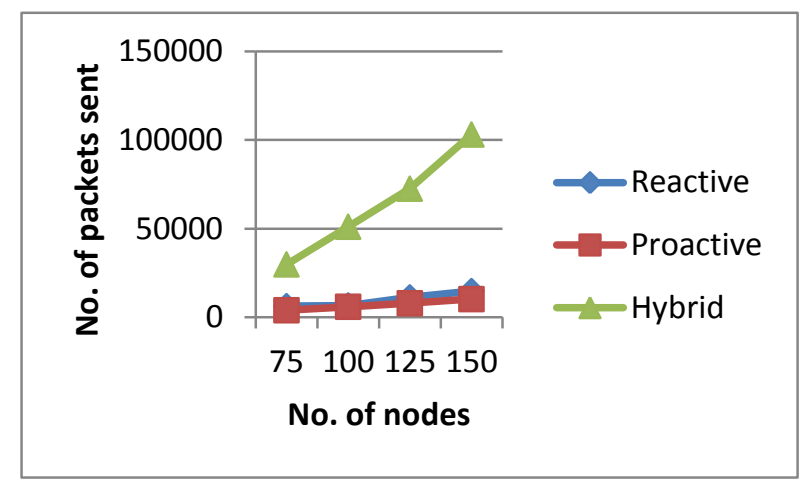

Figure1: Number of packets sent for reactive, proactive and hybrid routing protocol using different number of nodes

In figure1, we observe that in the hybrid routing protocol maximum number of packets are sent than the other two protocols. Also, the number of packets sent goes on increasing with increase in number of nodes. But the number of packets sent for proactive and reactive protocols are very less but are comparable to each other.

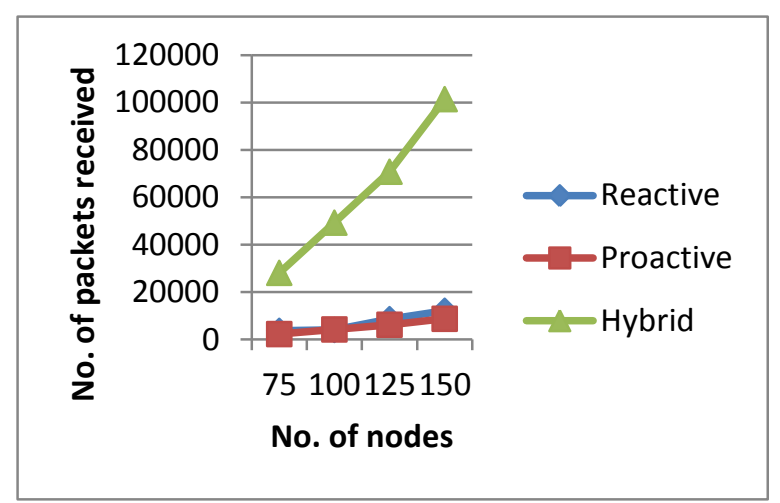

Figure2:Number of packets received for reactive, proactive and hybrid routing protocol using different number of nodes

In figure2,we observe that in the hybrid routing protocol maximum number of packets are sent than the other two protocols. Also, the number of packets sent goes on increasing with increase in number of nodes. But the number of packets sent for proactive and reactive protocols are very less but are comparable to each other.

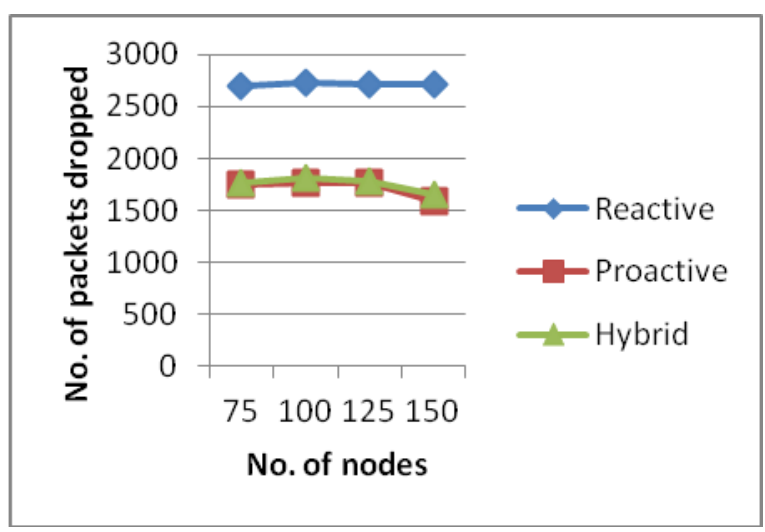

Figure3: Number of packets dropped for reactive, proactive and hybrid routing protocol using different number of nodes

In figure3, we observe that in case of reactive routing protocol, maximum number of packets are dropped. For proactive and hybrid routing protocols, the number of packets dropped are almost equal but are less in comparison to reactive routing protocol. 


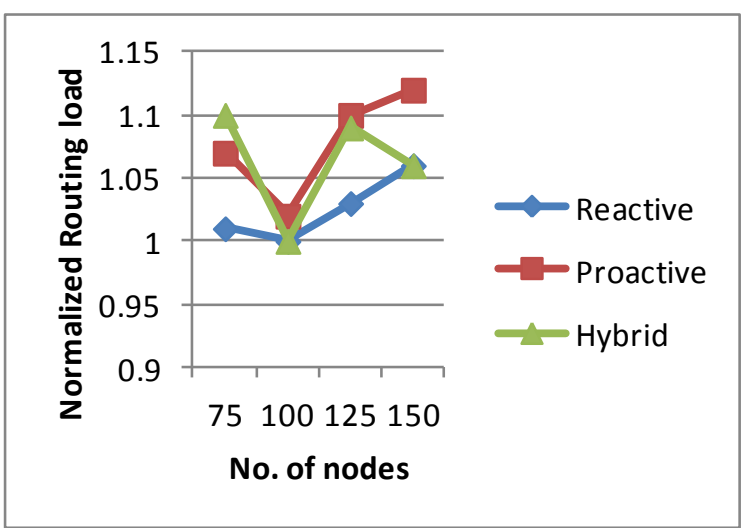

Figure4: Normalized Routing Load for reactive, proactive and hybrid routing protocol using different number of nodes

In figure 4,we observe that normalized Routing load for proactive routing protocol has more value as compared to reactive and hybrid protocols. Here, the reactive protocol has least value.

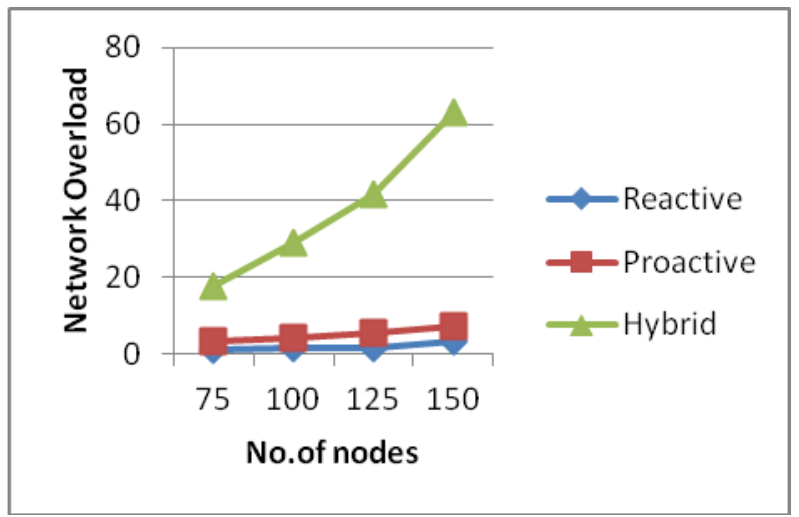

Figure5: Network overload for reactive, proactive and hybrid routing protocol using different number of nodes

In figure5,we observe that due to large number of packets in case of hybrid protocols, network overload is very high in comparison to reactive and proactive routing protocols. The value of network overload for reactive protocol is analogous to proactive protocol.

\section{CONCLUSION}

In this research paper, the whole analysis is based on proactive, reactive and hybrid routing protocols. Using different performance parameters these distinct protocols are compared by having different number of nodes. This simulation analysis is purely dependent on the parameters viz. number of packets sent, number of packets received, number of packets dropped, Normalized routing load and network overload. this paper concludes that the hybrid protocol provides better performance than the other two protocols. Among proactive and reactive routing protocols, reactive protocol is counted better due to minimum value of NRL. For future work, the protocols which are used can be altered and also the work can be extended using more number of nodes.

\section{REFERENCES}

[1] Aarti, D. S. (2013). Study of MANET: Characteristics, Challenges, Application and Security Attacks. International Journal of Advanced Research in Computer Science and Software Engineering, 252-257.

[2] AMANDEEP, G. K. (2012). PERFORMANCE ANALYSIS OF AODV ROUTING PROTOCOL IN MANETS. International Journal of Engineering Science and Technology (IJEST), 3620-3625.

[3] Jeroen Hoebeke, I. M. (2004), "An Overview of Mobile Ad Hoc", Journal of the Communications Network , 3 , pp.60-66.

[4] Kumkum Parmar, A. A. (2012). EFFECTIVE SESSION ESTABLISHMENT AND DATA TRANSMISSION IN MANET. IJREAS , 1323-1329.

[5] Mina Vajed Khiavi, S. J. (2012), "Performance Comparison of AODV, DSDV, DSR and TORA Routing Protocols in MANETs", International Research Journal of Applied and Basic Sciences , 3 (7), 1429-1436.

[6] Mohapatra, P., \& Krishnamurthy, S. (2005),ADHOC Networks Technologies and Protocols. Boston: Springer Science.

[7] Rai, R. K. (2012),"A Novel Review on Routing Protocols in MANETs", Undergraduate Academic Research Journal (UARJ), , 1 (1), 103-108.

[8] Swati Bhasin, A. G. (2012), "Comparison of AODV, OLSR and ZRP Protocols in Mobile Ad-hoc Network on the basis of Jitter",International Journal of Applied Engineering Research , 7.

[9] Verma, A. S. (2013). SIMULATION AND ANALYSIS OF AODV, DSDV, ZRP IN VANET. International Journal in Foundations of Computer Science \& Technology (IJFCST), 13-26.

[10] Vishal Sharma, H. S. (2012)," Performance evaluation of reactive routing protocols in MANET networks using GSM based voice traffic applications", International Journal for light and electron optics , 1-4 